\title{
Expression of Some Special Functions through $q$-Exponentials of the Nonadditive Statistical Mechanics
}

\author{
Leonardo S. Lima \\ Department of Physics, Federal Education Center for Technological Education of Minas Gerais, Belo Horizonte, Brazil \\ Email:1slima@cefetmg.br
}

How to cite this paper: Lima, L.S. (2020) Expression of Some Special Functions through $q$-Exponentials of the Nonadditive Statistical Mechanics. Journal of Modern Physics, 11, 81-90.

https://doi.org/10.4236/jmp.2020.111004

Received: November 28, 2019

Accepted: January 7, 2020

Published: January 10, 2020

Copyright (อ 2020 by author(s) and Scientific Research Publishing Inc. This work is licensed under the Creative Commons Attribution International License (CC BY 4.0).

http://creativecommons.org/licenses/by/4.0/

\begin{abstract}
Generalized $q$-exponentials functions are employed to make a generalization of complete and incomplete gamma functions. We obtain a generalization of this class of special functions which are very important in the fields of probability, statistics, statistical physics as well as combinatorics and we derive some of its properties. One gets that the generalized gamma function obtained whether approaches of the standard gamma function for a specific $q$ values such as $q=q_{0} \approx 0.9$ value suffering a large variation with the variation of $q$.
\end{abstract}

\section{Keywords}

$q$-Exponential Function, Special Function, Gamma Function

\section{Introduction}

The generalization of elementary functions such as exponential function in $q$-exponential has been proposed since long time ago [1]. However, more recently, another type of $q$-exponential has been introduced in the environment of the nonadditive statistical mechanics [2], where the theory has been proposed as a generalization of the standard Boltzmann-Gibbs statistical mechanics for the class of system that is non-ergotic. Many other $q$-representations were proposed such as the $q$-algebra [3], the $q$-Fourier transform [2] [4], the $q$-Dirac's delta function [5] [6], the $q$-Gaussian [5] [7] [8] and so on. Moreover, combinations of complex $q$-exponentials were used to make a new representation of the classical nonlinear oscillator [9], where a combination of complex $q$-exponentials have been of interest also for recent $q$-generalized Schrödinger and Dirac equations and other field equations [7]. In the non-additive statistical mechanics the 
entropy is described by the so called Tsallis entropy [2], previously introduced by Havrdat-Charvát [10] and later on by Daróczy [11], parameterized by an exponent $q$. The distribution that maximizes the Tsallis entropy under the constraint of the fixed energy generalizes the Boltzmann distribution through a generalized $q$-exponential function. Furthermore, in the nonadditive statistical mechanics, when the limit of the parameter of non-extensiveness $q$ is $q \rightarrow 1$, one recovers the standard exponential (and thus, the Gaussian or Boltzmann distribution). Many other classical equations (e.g. the Schrödinger equation) were generalized, replacing the classical exponential with the $q$-exponential. One example of non-ergodic systems that have been studied is system with long range interactions [12] [13] [14].

In addition, there is another definition of $q$-exponential given in combinatorial mathematics, where $q$-exponential is a $q$-analog of the standard exponential function namely, the eigenfunction of a $q$-derivative. There are many $q$-derivatives, for example, the classical $q$-derivative, the Askey-Wilson operator and so on [15]. Therefore, unlike the classical exponentials, $q$-exponentials are not unique. Moreover, it has been constructed rational expansions to $e^{x}$ by Németh and Newman in Ref. [16].

A special function very important is the gamma function $\Gamma(z), z \in \mathbb{C}$, $\Re e\{z\}>0$. This is an extension for the complex plane of the factorial function $n$ !, for $n \in \mathbb{N}$, with its argument shifted down by 1 , to real and complex numbers. Thus, the gamma function is defined for all complex numbers except for non positive integers. It raises as component in various probability distributions, and as such it is applicable in the fields of probability, statistics and statistical physics, as well as in combinatorics [17] [18] [19] [20] [21]. For instance, the well known Stirling formula depends on the factorial function as [22]

$$
\sqrt{2 \pi n}\left(\frac{n}{e}\right)^{n} \leq n ! \leq \sqrt{2 \pi n}\left(\frac{n}{e}\right)^{n} e^{(1 / 12 n)}
$$

Furthermore, this function if relate with the Shannon's entropy that gives an approach to the information measure

$$
\lim _{n \rightarrow \infty} \frac{1}{n} \ln \left[\frac{n !}{\left(p_{1} n\right) !\left(p_{2} n\right) ! \cdots\left(p_{k} n\right) !}\right]=-\sum_{k=1}^{k} p_{i} \ln p_{i} .
$$

The gamma function is extended by analytic continuation to all complex numbers except the non-positive integers (where the function has simple poles), yielding a meromorphic function which we call the gamma function. It has no zeroes and hence, the reciprocal gamma function $1 / \Gamma(z)$ is a holomorphic function. In fact, gamma function corresponds to Mellin transform [23] of the negative exponential function: $\Gamma(z)=\left\{\mathcal{M} e^{-x}\right\}(z)$.

The generalized gamma function can be applied to nonlinear systems (NL) class obeyed by the nonlinear equations. This has become an important subject in the mathematical physics in recent years due to their ability to explain several complex behaviors in the nature [24] [25]. Many areas of physics such as plasma 
physics and non-equilibrium have benefited from the study of NL equations [7]. However, it is well known that, in general, nonlinear problems are impossible to solve analytically. The essential difference is that linear systems can be broken down into parts while the nonlinear not. When the parts of a system interfere, either cooperate or compete. The interactions are nonlinear and hence, the principle of superposition fails [26]. Within the realm of physics, the nonlinearity is vital for many areas, for instance, for the superconductivity and Josephson junctions arrays [26].

In this work, we propose the representation of some special functions such as the gamma function in terms of $q$-exponentials functions of nonadditive statistical mechanics. The plan of this paper is the following. In Section 2, we represent the generalized gamma function expressed in terms of $q$-exponential functions. In Section 3, we use another representation for the $q$-exponential in the literature to represent the generalized gamma function. In the last section, Section 4 , we present our conclusions and final remarks.

\section{Generalized Gamma Function}

The generalized gamma function, defined using the $q$-exponential distribution, is defined by the integral

$$
\Gamma_{q}(z+1)=\int_{0}^{\infty} x^{z} e_{q}^{x} \mathrm{~d} x
$$

where $q \in(0,1]$ and $z \in \mathbb{C}$ and $\mathfrak{R}\{\{z\}>0$. In the limit $q \rightarrow 1$, we have $\Gamma_{q}(z)=\Gamma_{1}(z)=\Gamma(z)$ and $\Gamma(n)=(n-1) !$ for $n \in \mathbb{N}$ and $\Gamma(z+1)=z \Gamma(z)$ for $z \in \mathbb{C}$.

Since $e_{q}^{x-1} \ll x^{z-1}$, when $x$ is positive and $x \in(0,1]$, we can write

$$
\left|\int_{0}^{1} e_{q}^{x} x^{z-1} \mathrm{~d} x\right|<\left|\int_{\epsilon}^{1} x^{z-1} \mathrm{~d} x\right|=\frac{1}{Z}-\frac{\epsilon^{z}}{Z}
$$

and, for $x>0$ the integral is limited for $1 / x$.

By making $x$ fixed and $\epsilon$ decreasing the value of integral increases monotonically, i.e.

$$
\begin{gathered}
\int_{0}^{1} e_{q}^{x} x^{z-1} \mathrm{~d} x=\lim _{\epsilon \rightarrow 0} \int_{\epsilon}^{1} e_{q}^{x} x^{z-1} \mathrm{~d} x \\
\exists \forall x>0 .
\end{gathered}
$$

$e_{q}^{i x}$ presents the properties $\left[e_{q}^{i x}\right]^{*}=\left[e_{q}^{-i x}\right]$. The $q$-exponential function is a deformation of the standard exponential function using the real parameter $q$ [27] [28]

$$
e_{q}^{x}= \begin{cases}{[1+(q-1) x]^{\frac{1}{q-1}},} & -\infty<x \leq 0, \\ {[1+(1-q) x]^{\frac{1}{1-q}},} & 0 \leq x<\infty .\end{cases}
$$

The inverse of the $q$-exponential function is the $q$-logarithm function, $\ln _{q}(x)$, defined as 


$$
\ln _{q}(x)= \begin{cases}\frac{x^{q-1}-1}{q-1}, & 0<x \leq 1, \\ \frac{x^{1-q}-1}{1-q}, & 1 \leq x<\infty .\end{cases}
$$

The formulae in Equation (6) hold true only for $q \in(0,1]$. In this interval $x$ and $q$ are mathematically independent. The complete definitions can be given in two equivalent ways: one either changes the full expression of the $q$-deformed functions appropriately and uses the interval $q \in(0,1]$ only as in the equation above or one can consider a unique expression and change the deformation parameter interval as [28]

$$
\begin{aligned}
& e_{q}^{x}=[1+(1-q) x]^{\frac{1}{q-1}} \begin{cases}-\infty<x \leq 0, & q \in[1,2), \\
0 \leq x<\infty, & q \in(0,1] .\end{cases} \\
& \ln _{q}(x)=\frac{x^{1-q}-1}{1-q} \begin{cases}0<x \leq 1, & q \in[1,2), \\
1 \leq x<\infty, & q \in(0,1] .\end{cases}
\end{aligned}
$$

The parameter $q$ represents the degree of non-additivity. Thus, solving the integral, Equation (3) and using the definition of $q$-exponential, we obtain the generalized gamma function given as

$$
\begin{aligned}
& \Gamma_{q}(p+1) \\
& =\frac{p(p-1)(p-2)(p-3) \times \cdots \times[p-(p-1)]}{(2-q)(3-2 q)(4-3 q)(5-4 q) \times \cdots \times[p+2-(p+1) q]} \int_{0}^{\infty}\left(e_{q}^{-x}\right)^{(p+2)(1-q)+q} \mathrm{~d} x,
\end{aligned}
$$

where $\Gamma(p+1)=p !$ for $p \in \mathbb{N}$ and $\Gamma(z+1)=z \Gamma(z), \quad z \in \mathbb{C}$. Therefore, using the standard factorial function, we have the recurrence relation obeyed for the generalized gamma function given by

$$
\Gamma_{q}(z+1)=\frac{z \Gamma(z)}{\prod_{j=1}^{p}[j+2-(j+1) q]},
$$

Consequently, we can obtain the expression for the $q$-factorial, $[p]_{q} !$ as

$$
[p]_{q} !=\frac{p !}{\prod_{j=1}^{p}[j+2-(j+1) q]}
$$

where $p \in \mathbb{N}$.

We also obtain the generalized incomplete gamma functions

$$
\begin{aligned}
& \Gamma_{q}(a, x)=\int_{x}^{\infty} z^{a-1} e_{q}^{z} \mathrm{~d} z . \\
& \gamma_{q}(a, x)=\int_{0}^{x} z^{a-1} e_{q}^{z} \mathrm{~d} z,
\end{aligned}
$$

with $\mathfrak{R} e(a)>0$, where

$$
\Gamma_{q}(a, x)+\gamma_{q}(a, x)=\Gamma_{q}(a) .
$$

In addition, we have the following generalized functions that if relate with the 
incomplete generalized gamma function

$$
\operatorname{erfc}_{q}(x)=\frac{1}{\sqrt{\pi}} \gamma_{q}\left(1 / 2, x^{2}\right)
$$

that is the generalized complementary error function, besides

$$
\mathrm{E}_{q n}(x)=\int_{1}^{\infty} \frac{e_{q}^{-x t}}{t^{n}} \mathrm{~d} t
$$

where we define the generalized exponential integral function $\mathrm{E}_{q 1}(x)=-\mathrm{E}_{q} i(-x)$ as

$$
\mathrm{E}_{q 1}(x)=\int_{-\infty}^{x} \frac{e_{q}^{t}}{t} \mathrm{~d} t
$$

In Figure 1 we plot the generalized gamma function $\Gamma_{q}(z)$ for $q$ value $q=0.9$ and for standard gamma function $\Gamma(z)$ (that corresponds to $q=1$ case. The graphic of $\Gamma_{q}(z)$ changes a lot with $q$ value as showed in Figure 2. This is due to the fact the $q$-exponential function to represent a family of functions (one for each value of $q$ within interval $(0,1)$ where the case $q=1$ (the standard exponential function, $e^{x}$ ) corresponds to only one function of these class of $q$-exponential functions. We get that for the particular case $q=0.9$, the $q$-gamma function exhibits a behavior nearest to standard exponential than $q$-exponentials defined for other $q$ values.

By using the definition of $q$-exponential, we obtain the generalized incomplete $q$-gamma function given as

$$
\Gamma_{q}(a, x)=\frac{x^{a-1}}{2-q}\left(e_{q}^{-x}\right)^{2-q}+\frac{a-1}{2-q} \Gamma_{q}(a-1, x)
$$

and

$$
\gamma_{q}(a, x)=\frac{x^{a-1}}{q-2}\left(e_{q}^{-x}\right)^{2-q}+\frac{a-1}{2-q} \gamma_{q}(a-1, x)
$$

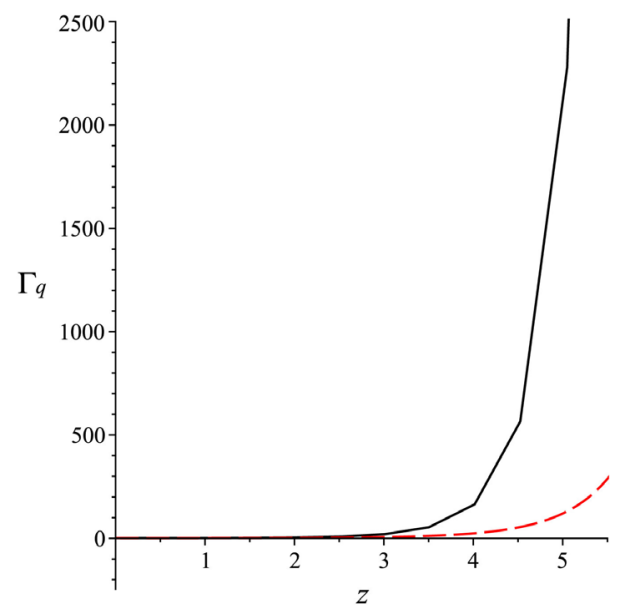

Figure 1. Plot of the generalized gamma function $\Gamma_{q}(z)$ for $q$ value $q=0.9$ and for standard gamma function $\Gamma(z)$ (that corresponds to $q=1$ case. 


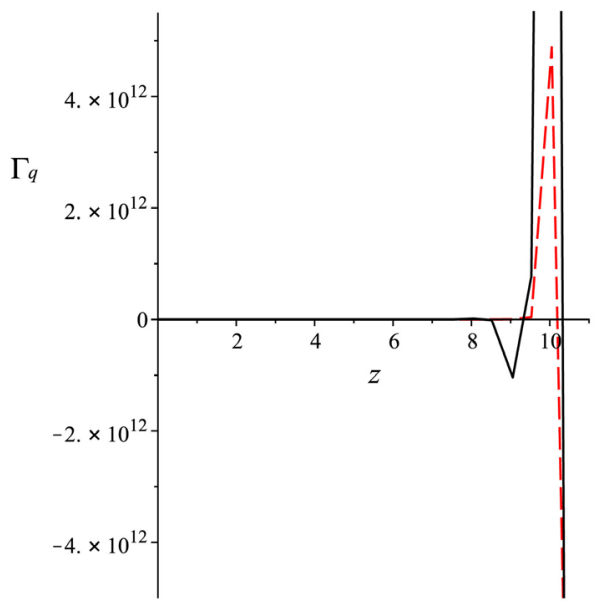

Figure 2. Plot of the generalized gamma function $\Gamma_{q}(z)$ for $q$ values $q=0.91$ (red-dashed-line) and $q=0.89$ (black-solid-line). The standard gamma function $\Gamma(z)$ case corresponds to $q=1$ case.

In addition, we also obtain the generalized exponential integral function as

$$
\mathrm{E}_{q 1}(x)=\frac{1}{2-q}\left(e_{q}^{x}\right)^{\frac{2-q}{1-q}} .
$$

Finally, we have the generalized logarithm integral function $\operatorname{li}_{q}(x)=\mathrm{E}_{q} i\left(\ln _{q}(x)\right)$, given by

$$
\mathrm{li}_{q 1}(x)=\int_{0}^{x} \frac{\mathrm{d} t}{\ln _{q}(t)} .
$$

Consequently, we obtain $\operatorname{li}_{q}(x)$ given as

$$
\mathrm{li}_{q 1}(x)=\int_{0}^{x} \frac{\mathrm{d} t}{\ln _{q}(t)}=(1-q) \int_{0}^{x} \frac{\mathrm{d} t}{t^{1-q}-1}=-\int_{0}^{x} \mathrm{~d} t \sum_{n=0}^{\infty}\left(t^{1-q}\right)^{n}=-\sum_{n=0}^{\infty} \frac{x^{n(1-q)+1}}{n(1-q)+1},
$$

where $|x|<1$.

\section{Another Representation}

Another type of $q$-exponential is defined using the following series expansion [1]

$$
e_{q}^{-x}=\sum_{n=0}^{\infty} \frac{(-x)^{n}}{[n]_{q} !}=\sum_{n=0}^{\infty} \frac{(-x)^{n}(1-q)^{n}}{(q ; q)_{n}},
$$

where $[n]_{q}$ ! is the $q$-factorial [29]

$$
\begin{aligned}
{[n]_{q} ! } & =[1]_{q}[2]_{q} \cdots[n-1]_{q}[n]_{q} \\
& =\frac{1-q}{1-q} \frac{1-q^{2}}{1-q} \cdots \frac{1-q^{n-1}}{1-q} \frac{1-q^{n}}{1-q} \\
& =1 \cdot(1+q) \cdots\left(1+q+\cdots+q^{n-2}\right)\left(1+q+\cdots+q^{n-1}\right) \\
& =\frac{(q ; q)_{n}}{1-q^{n}},
\end{aligned}
$$

where we use the $q$-bracket given by 


$$
[n]_{q}=\frac{1-q^{n}}{1-q}
$$

In addition, we have the $q$-Pochhammer symbol given as [30] [31]

$$
(q ; q)_{n}=\left(1-q^{n}\right)\left(1-q^{n-1}\right) \cdots(1-q)
$$

The graphic of $y=e_{q}^{-x}$, given by Equation (23), for $q$ value of $q=0.9$ is given in Figure 3. The black-solid-line corresponds to series in Equation (23) until $n=10$. The red-dashed-line correspond to sum until $n=100$. So, for $n>100$, the behavior of graphic does not change a lot (for $n \rightarrow \infty$ ).

The generalized gamma function given by the integral

$$
\Gamma_{q}(z+1)=\int_{0}^{\infty} x^{z} e_{q}^{-x} \mathrm{~d} x,
$$

In Figure 4, we plot the generalized gamma function $\Gamma_{q}(z)$ for $z \in \mathbb{N}$ and $q$ value as $q=0.9$. The behavior of the graphic changes with $q$ as in the before case. However, we have here, $q \in \mathbb{R}$ and $z \in \mathbb{N}$ and $\mathfrak{R} e\{z\}>0$. Remembering that here, this $q$-exponential is not that defined in the nonadditive statistical mechanics. Therefore, we obtain the integral Equation (27) as (Figure 4)

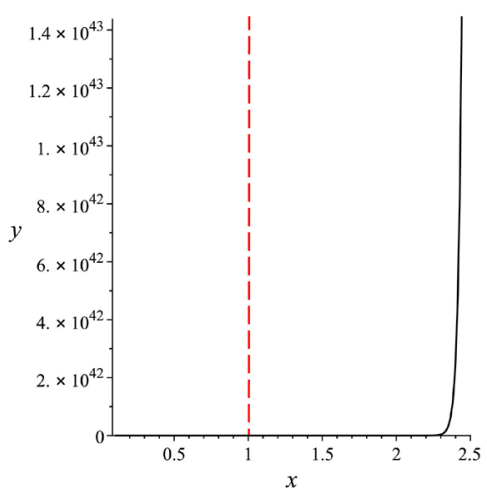

Figure 3. Plot of $y=e_{q}^{-x}$, given by Equation (23), for $q$ value of $q=0.9$. The black-solid-line corresponds to series in Equation (23) until $n=10$. The red-dashed-line correspond to sum until $n=100$.

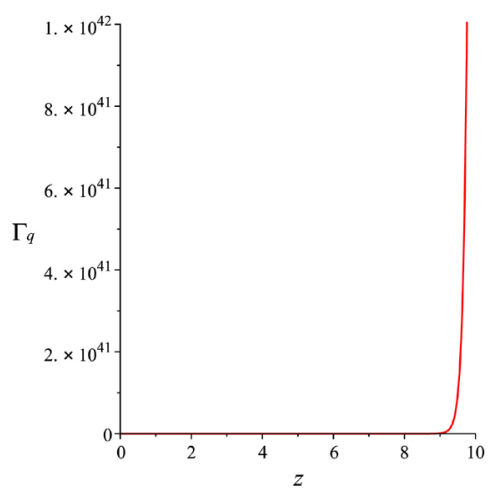

Figure 4. Graphic of generalized gamma function $\Gamma_{q}(z)$ for integer $z$, and $q=0.9$. The sum in series given by Equation (23) is for $n=100$. The $\Gamma_{q}$ axis has been rescaled as $\Gamma_{q} \rightarrow \Gamma_{q} / 7.773783455 \times 10^{40005}$. 


$$
\Gamma_{q}(z+1)=\int_{0}^{\infty} x^{z} e_{q}^{-x} \mathrm{~d} x=\sum_{n=0}^{\infty} \frac{(-1)^{n}}{[n]_{q} !} \int_{0}^{\infty} \frac{\mathrm{d} x}{x^{n+z}}
$$

where the integration contour $C$ in the integral above is represented in Figure 5; and $z<-n-1$. The integral above can be solved using the residue technique as

$$
\oint_{C} \frac{\mathrm{d} z}{z^{n+\xi}}=\int_{\epsilon}^{\infty} \frac{\mathrm{d} x}{x^{n+z}}+\int_{\infty}^{\epsilon} \frac{\mathrm{d} x}{x^{n+z}}+\int_{C^{\prime}} \frac{\mathrm{d} z}{z^{n+\xi}},
$$

where $z=\epsilon e^{i \theta}, \mathrm{d} z=i \epsilon e^{i \theta} \mathrm{d} \theta$, where we have taken the limit $\epsilon \rightarrow 0$ and thus, from the above integral

$$
\left[e^{2 \pi i(n+\xi)}-1\right] \int_{0}^{\infty} \frac{\mathrm{d} x}{x^{n+z}}=\left.\frac{2 \pi i}{(n+\xi+1) !} \frac{\mathrm{d}^{n+\xi+1}}{\mathrm{~d} z^{n+\xi+1}}\left[\left(z-z_{0}\right)^{n+\xi} \frac{1}{z^{n+\xi}}\right]\right|_{z_{0}=0}=0
$$

which cancels for $\forall z \mid z<-n-1$. For other $z$ values the integral of the generalized gamma function diverges. However, for the incomplete gamma function

$$
\gamma_{q}(z+1, x)=\int_{0}^{x} t^{z} e_{q}^{-t} \mathrm{~d} t=\sum_{n=0}^{\infty} \frac{(-1)^{n}}{[n]_{q} !} \int_{0}^{x} t^{n+z} \mathrm{~d} t=\sum_{n=0}^{\infty} \frac{(-1)^{n}}{[n]_{q} !} \frac{x^{n+z+1}}{n+z+1},
$$

The integral of the incomplete generalized gamma function converges $\forall n, z$ values. Because of the complicated behavior of the derivative of the $q$-exponential function, the generalized gamma function here does not obey the property $\Gamma(z+1)=z \Gamma(z)$ obeyed by the standard gamma function.

For the generalized exponential integral function, we obtain

$$
\mathrm{E}_{q} i(x)=\int_{x}^{b} \frac{e_{q}^{-t}}{t} \mathrm{~d} t=\sum_{n=0}^{\infty} \frac{(-1)^{n-1}}{[n]_{q} !} \int_{x}^{b} t^{n-1} \mathrm{~d} t
$$

where $b \in \mathbb{R}$. However for $b \rightarrow \infty$ the integral above diverges. The same behavior we have for the generalized integrals Equation (16).

\section{Final Remarks}

In summary, we have proposed a generalization of the complete and uncomplete gamma functions using $q$-exponential functions. This subject can be of interest

$\Lambda \operatorname{Im} z$

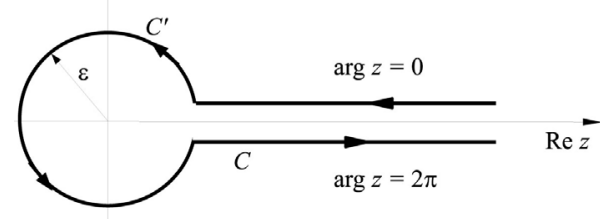

Figure 5. Contour for the calculation of the integral in the Equation (29). 
in many branches of physics due to the many phenomena associated with the nonlinearity. Recently, a connection between generalized functions of the nonadditive statistical mechanics framework and nonlinear equations has been investigated. In literature, were proposed other deformations of the $\Gamma$-function in terms of deformed factorials [32], where the $q$-factorial is $x ! \bigotimes_{q}=e_{q}^{\sum_{i=1}^{x} \ln (i)}=\Gamma_{q}(x+1)$. These definitions are different from the proposed here.

\section{Acknowledgements}

This work was partially supported by CNPq (National Council for Scientific and Technological Development.

\section{Conflicts of Interest}

The author declares no conflicts of interest regarding the publication of this paper.

\section{References}

[1] Exton, H. (1983) Q-Hypergeometric Functions and Applications. Halstead Press, New York

[2] Tsallis, C. (1988) Journal of Statistical Physics, 52, 479-487. https://doi.org/10.1007/BF01016429

[3] Tsallis, C. (2009) Introduction to Nonextensive Statistical Mechanics. Springer, Santa Fé, New Mexico.

[4] Jauregui, M. and Tsallis, C. (2011) Physics Letters A, 375, 2085-2088. https://doi.org/10.1016/j.physleta.2011.04.014

[5] Tsallis, C. (2012) International Journal of Bifurcation and Chaos, 22, Article ID: 1230030. https://doi.org/10.1142/S0218127412300303

[6] Jauregui, M. and Tsallis, C. (2010) Journal of Mathematical Physics, 51, Article ID: 063304. https://doi.org/10.1063/1.3431981

[7] Nobre, F.D., Rego-Monteiro, M.A. and Tsallis, C. (2012) Europhysics Letters, 97, Article ID: 41001. https://doi.org/10.1209/0295-5075/97/41001

[8] Nobre, F.D., Rego-Monteiro, M.A. and Tsallis, C. (2011) Physical Review Letters, 106, Article ID: 140601. https://doi.org/10.1103/PhysRevLett.106.140601

[9] Lima, L.S. (2017) The European Physical Journal B, 90, 180. https://doi.org/10.1140/epjb/e2017-80255-8

[10] Havrda, J. and Charvát, F. (1967) Kybernetika, 3, 30-35.

[11] Daróczy, Z. (1970) Information and Computation, 16, 36-51. https://doi.org/10.1016/S0019-9958(70)80040-7

[12] Cirto, L.J.L., Lima, L.S. and Nobre, F.D. (2015) Journal of Statistical Mechanics, 4, P04012. https://doi.org/10.1088/1742-5468/2015/04/P04012

[13] Cirto, L.J.L., Assis, V.R.V. and Tsallis, C. (2014) Pysica A, 393, 286-296. https://doi.org/10.1016/j.physa.2013.09.002

[14] Nobre, F.D. and Tsallis, C. (2003) Physical Review E, 68, Article ID: 036115. https://doi.org/10.1103/PhysRevE.68.036115

[15] Gasper, G. and Rahman, M. (2004) Basic Hypergeometric Series. Cambridge Uni- 
versity Press, Cambridge. https://doi.org/10.1017/CBO9780511526251

[16] Komarov, M.A. (2018) (2019) Journal of Approximation Theory, 240, 126-128. https://doi.org/10.1016/j.jat.2018.10.001

[17] Dar, S.A. and Paris, R.B. (2019) Journal of Computational and Applied Mathematics, 348, 237. https://doi.org/10.1016/j.cam.2018.08.045

[18] Wang, X. (2016) Integral Transforms and Special Functions, 27, 347. https://doi.org/10.1080/10652469.2015.1128432

[19] Abd-Elhameed, W.M. (2015) Integral Transforms and Special Functions, 26, 586-599. https://doi.org/10.1080/10652469.2015.1029924

[20] Brychkov, Y.A. and Saad, N. (2014) Integral Transforms and Special Functions, 25, 111-123. https://doi.org/10.1080/10652469.2013.822207

[21] Bibby, B.M. and Vth, M. (2011) Statistics and Probability Letters, 81, 884. https://doi.org/10.1016/j.spl.2010.12.019

[22] Dutka, J. (1991) Archive for History of Exact Sciences, 43, 225-249. https://doi.org/10.1007/BF00389433

[23] Whittaker, E.T. and Watson, G.N. (1996) A Course of Modern Analysis. Cambridge University Press, Cambridge. https://doi.org/10.1017/CBO9780511608759

[24] Habib, G. and Kerschen, G. (2016) Physica D, 332, 1-8. https://doi.org/10.1016/j.physd.2016.06.001

[25] Fernández-García, S., Krupa, M. and Clément, F. (2016) Physica D, 332, 9-22. https://doi.org/10.1016/j.physd.2016.06.002

[26] Strogatz, S.H. (1994) Nonlinear Dynamics and Chaos. Cambridge University Press, Cambridge.

[27] Umarov, S., Tsallis, C. and Steinberg, S. (2008) Milan Journal of Mathematics, 76, 307-328. https://doi.org/10.1007/s00032-008-0087-y

[28] Oikonomou, T. and Gagci, G.B. (2010) Physics Letters A, 374, 2225-2229. https://doi.org/10.1016/j.physleta.2010.03.038

[29] McIntosh, R.J. (1999) The Ramanujan Journal, 3, 205. https://doi.org/10.1023/A:1006949508631

[30] Guidottia, P. and Shaob, Y. (2017) Nonlinear Analysis, 150, 114. https://doi.org/10.1016/j.na.2016.11.003

[31] Schmidt, M.D. (2017) Combinatorial Identities for Generalized Stirling Numbers Expanding $f$-Factorial Functions and the $f$-Harmonic Numbers.

[32] Oikonomou, Th. (2007) Physica A, 386, 119-134. https://doi.org/10.1016/j.physa.2007.08.025 